\title{
Empleo del storytelling y las narrativas en primera persona en la comunicación de Las Kellys como referente para las ONGs
}

\author{
The use of storytelling and first-person narratives in \\ communication by Las Kellys as a reference for NGOs
}

\author{
Cristóbal Fernández-Muñoz; Miguel Tomé-Caballero
}

Cómo citar este artículo:

Fernández-Muñoz, Cristóbal; Tomé-Caballero, Miguel (2020). “Empleo del storytelling y las narrativas en primera persona en la comunicación de Las Kellys como referente para las ONGs". Profesional de la información, v. 29, n. 3, e290325.

https://doi.org/10.3145/epi.2020.may.25

Artículo recibido el 11-11-2019

Aceptación definitiva: 19-03-2020
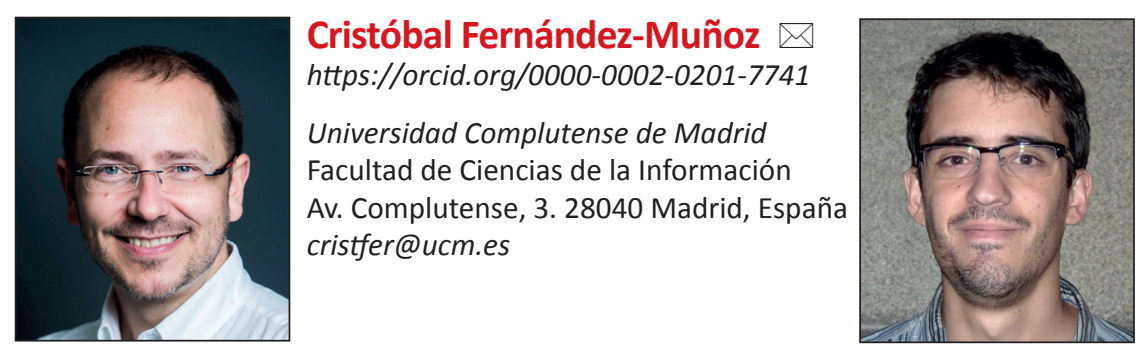

\begin{abstract}
Miguel Tomé-Caballero
https://orcid.org/0000-0003-1389-3277

Universidad Complutense de Madrid Facultad de Ciencias de la Información Av. Complutense, 3. 28040 Madrid, España migtome@ucm.es
\end{abstract}

\section{Resumen}

En el contexto de la crisis socioeconómica de los últimos años en España en el que las ONGs y los movimientos sociales han tenido que enfrentarse a importantes retos y oportunidades de comunicación, este trabajo analiza el caso de Las Kellys, la organización de camareras de piso creada en 2016, que ha utilizado el storytelling en sus acciones de Relaciones Públicas con los medios para trasladar con éxito a la sociedad sus reivindicaciones de mejora de sus derechos laborales. Se ha combinado una metodología cualitativa y cuantitativa para evaluar los medios comunicativos empleados y el impacto de sus acciones, realizando aportaciones que pueden ser de utilidad para maximizar la eficacia comunicativa de otras organizaciones, especialmente de las ONGs, a través de las narrativas en primera persona, con un lenguaje cercano y coloquial que las dotan de plena autenticidad.

\section{Palabras clave}

Comunicación; Movimientos sociales; ONG; Storytelling; Impacto mediático; Relaciones Públicas; Prensa; Las Kellys.

\begin{abstract}
In the context of the socioeconomic crisis of recent years in Spain, in which NGOs and social movements have had to face significant communication challenges and opportunities, this paper analyzes the case of Las Kellys, an organization for hotel maids created in 2016 that has used storytelling in its public relations actions with the media to successfully transfer to society its claims to improve their labor rights. Qualitative and quantitative methodologies are combined to evaluate the communication tools used and the impact of their actions, providing useful insights to maximize the communicative effectiveness of other organizations, especially NGOs, through first-person narratives, with fully authentic, familiar and colloquial language.
\end{abstract}

Keywords

Communication; Social movements; NGOs; Storytelling; Media impact; Public Relations; Press; Las Kellys. 


\section{Introducción}

Desde la década de 2000 se planteó a nivel global la necesidad de exigir a las organizaciones no gubernamentales un mayor rigor en su trabajo, una actitud crítica con las injusticias sociales y unas nuevas pautas éticas en su conducta que situaran a la persona como eje de sus actuaciones en una sociedad cambiante (Gómez-Gil, 2005). Los escándalos que hicieron protagonistas a varias ONGs españolas, ocasionaron críticas sobre el funcionamiento, financiación y control de estas entidades que supuso una pérdida de credibilidad y la necesidad de incrementar su transparencia (Gálvez-Rodríguez; Caba-Pérez; López-Godoy, 2009). Adicionalmente, la crisis económica en la década posterior provocó que las ONGs en España se hayan enfrentado a importantes retos de comunicación, sintiéndose incapaces en muchas ocasiones de implicar a la ciudadanía en sus causas sociales y viéndose afectadas por un contexto de recesión que ha reducido el número de socios y, en consecuencia, sus fondos económicos (Arroyo-Almaraz; Baladrón-Pazos; Martín-Nieto, 2013). Por su parte, los sindicatos como actores tradicionales de movilización social han tenido un peso específico decreciente cuando se ha producido en el mismo período una revitalización de nuevos movimientos sociales que han apostado por un estilo de comunicación renovado y que ha logrado altas tasas de movilización ciudadana. El 15M, las plataformas antidesahucio, la defensa de pensiones dignas o el movimiento feminista son algunas de las luchas que han salido a la calle y han cobrado una notoriedad destacada entre la opinión pública española. Ante el enorme respaldo social de nuevos actores, los sindicatos son conscientes de que pierden el liderazgo en la movilización y corren el riesgo de desvincularse definitivamente de las demandas de una sociedad cada vez más precarizada. Como señala Marí-Sáez (2017), desde el año 2010 hasta la actualidad, la comunicación para el desarrollo y el cambio social se ha expandido en España con una rapidez que contrasta con el olvido y marginación que históricamente ha tenido, hasta convertirse en un fenómeno de moda.

Es en este contexto histórico donde se constituye, a comienzos de 2016, la asociación Las Kellys: un movimiento creado por camareras de piso (mujeres de la limpieza de hoteles) que se organizaron para reclamar sus derechos laborales.

El trabajo de las camareras de piso es un empleo feminizado y de duro desempeño diario. La limpieza de habitaciones implica estar

"un 85\% de la jornada laboral diaria en posiciones perjudiciales para el cuerpo" (Mantovano, 2015, p. 12).

Estas sobrecargas ocasionan lesiones físicas graves, principalmente en la zona lumbar y en el túnel carpiano. Según el informe de Jiménez-Fernández et al. (2010), casi el 50\% de las camareras de piso tienen lesiones en la zona lumbar.

El trabajo conlleva además unos altos niveles de estrés, muchas veces incrementado por los malos tratos hacia ellas de gobernantas, jefes o clientes. El $96 \%$ de las camareras sufre síntomas de ansiedad grave o leve y el 28,45\% presenta síntomas de depresión grave (Mantovano, 2015).

Las condiciones del empleo suelen ir asociadas a la temporalidad, dado que la duración de los contratos depende de los flujos turísticos y por tanto gran parte de las camareras de piso están contratadas a tiempo parcial o como fijas discontinuas.

La situación es peor cuando el servicio de limpieza del hotel está externalizado en empresas multiservicios, conocidas como ETT. Este tipo de empresas no están obligadas a conservar las condiciones laborales de los convenios colectivos del hotel. En cambio,

"crean su propio convenio con el que se benefician tanto ellas como las empresas que contratan sus servicios" (Jarque-Balaguer, 2018, p. 22).

De esta manera se hace una rebaja de categoría profesional -las empleadas pasan de camareras de piso a peones o limpiadoras- y pueden pagarlas unos salarios notablemente inferiores del que les correspondería si estuviesen contratadas bajo el convenio colectivo -hasta 300 o 400 euros menos al mes- (Cañada, 2015). Además, el nivel de trabajo exigido es mayor, teniendo que limpiar muchas más habitaciones en el mismo tiempo y con el mismo resultado.

Con el objetivo de dignificar las condiciones laborales, la Unión Internacional de los Trabajadores de la Agricultura, la Alimentación y la Hostelería (UITA) lanzó en 2013 en varios países una campaña que llevaba como nombre Arreglen mi puesto de trabajo. En España, la campaña se concretó en unos textos redactados por el periodista Ernest Cañada:

- artículo publicado el 30 de julio de 2014 en el periódico El país bajo el título "Las que limpian los hoteles" (Cañada, 2014);

- libro publicado en 2015 Las que limpian los hoteles. Historias ocultas de la precariedad laboral (Cañada, 2015).

A raíz de las entrevistas realizadas para estas publicaciones, varias de las mujeres consultadas decidieron crear en 2014 un grupo de Facebook de camareras de piso en el que poder compartir las preocupaciones de sus trabajos. Algunas de ellas consideraron que era necesario visibilizar la situación públicamente y por ello en 2016 se constituyó oficialmente la asociación Las Kellys, nombre que hacía referencia al título del artículo de Cañadas ("Las que limpian los hoteles"). La organización se fue ampliando y en el momento de realizar esta investigación tiene presencia en once delegaciones españolas: Madrid, Barcelona, Lanzarote, Gran Canaria, Fuerteventura, Tenerife, Benidorm, Cádiz, Alicante, Asturias y Mallorca.

Como resumen de sus reivindicaciones, estas mujeres propusieron la Ley Kelly, una serie de demandas que desde el origen del movimiento han estado publicadas en su blog.

https://laskellys.wordpress.com 
La Ley fue defendida en noviembre de 2017 en una ponencia pública en la Comisión de Peticiones del Parlamento Europeo, donde varias representantes de Las Kellys exigieron que se cumpliese la normativa laboral en el sector de las camareras de piso y se impidiesen las externalizaciones en las cadenas hoteleras. Esa situación genera la cuestión de qué estrategias de comunicación habían empleado estas mujeres -en muchos casos faltas de estudios y de formación- para haber llegado hasta allí y si alguna de dichas estrategias puede servir de referencia para departamentos de Comunicación y Relaciones Públicas de otras organizaciones.

\section{Retos y oportunidades de la comunicación de las ONGs y los movimientos sociales}

Numerosos investigadores (Erro-Sala, 2003; Balas-Lara, 2008; 2012; Jerez-Novara; Sampedro; López-Rey, 2008; Pagola-Carte, 2009; Santolino-Prieto, 2010; Martínez-Osés, 2011; Nos-Aldás; Iranzo; Farné, 2012; Barranquero-Carretero, 2014; Marín-Dueña; Gallardo-Vera, 2014; Nos-Aldás; Santolino-Prieto, 2015; Gómez-Pérez et al., 2019) coinciden en que las ONGs tienen un problema en su comunicación y que en gran parte se debe a que ni siquiera conocen el propósito de lo que comunican. El sociólogo Javier Erro-Sala fue pionero en este análisis, planteando que la comunicación de la mayoría de ONGs presenta una dicotomía: quieren transformar la realidad pero también tienen que sobrevivir económicamente, y detrás de estos objetivos hay sendas estrategias comunicativas que en muchos casos son contradictorias entre sí.

Pagola-Carte (2009) plantea el problema en la misma línea: las ONGs suelen llevar a cabo programas de cooperación cuya complejidad choca con el estilo de comunicación rápido y sencillo que parece ser necesario para atraer la atención de los medios y de un gran número de ciudadanos. Esto dificulta a sus departamentos de comunicación colocar su mensaje de la manera que les gustaría a través de acciones eminentemente de Relaciones Públicas.

El estilo comunicativo resultante de adecuar el mensaje a la rapidez de los medios es lo que Erro-Sala (2003) denominó "comunicación mercadeada", la cual tiene como principal objetivo la obtención de donaciones económicas. Según Benet (2003), dicho estilo está formado por comunicados simples y "legibles" para que se puedan comprender de manera rápida a pesar de la distancia cultural entre donantes y receptores de ayuda.

Gómez-Pérez et al. opinan que, intentando ser accesibles, las ONGs utilizan

"narrativas desproblematizadoras centradas en los resultados y en las soluciones lineales" (Gómez-Pérez et al., 2019, p. 78).

El problema de esto es que se corre el riesgo de transmitir la idea de que

"las desigualdades, la pobreza, los desastres naturales, etc., tienen solución por medio del dinero" (Díez-Rodríguez, 2002, p. 134).

En la misma línea, se genera la percepción errónea en la ciudadanía sobre los fines perseguidos por las ONGs y que se llegue creer que siempre que quieren comunicar algo lo hacen con la finalidad de pedir dinero (González-Luis, 2006).

En todo caso, Santolino-Prieto (2010) expone que la simplicidad del mensaje transmitido tiene como resultado que la distancia entre lo que las ONGs hacen y lo que las ONGs comunican sea enorme. Pese a ser entidades capaces de manejarse en debates políticos de elevada complejidad técnica, estos diálogos no se suelen trasladar a sus comunicaciones públicas. La única excepción que Santolino-Prieto señala son las organizaciones ecologistas, quienes sí que

"han logrado asentar en el imaginario colectivo la idea de que son necesarios cambios globales" (Santolino-Prieto, 2010, p. 231),

siendo algo que otras entidades tal vez no han sido capaces de generar en torno a otros temas como la desigualdad o la injusticia social. Así pues, se hace importante establecer relieves y peculiaridades singulares y la especialización ejecutoria de una determinada temática para que los medios, cuando informen sobre un asunto relacionado con esa cuestión, recurran a aquellos grupos que están en el seno de la sociedad reconocidos como agrupaciones especializadas en el asunto (Castillo-Esparcia, 2007).

Esta situación ha empezado precisamente a cambiar en la última década puesto que la relación con los medios ha ido variando.

"Las estrategias con medios de comunicación pasan cada vez más por aportar contenidos de calidad y contrastados a los profesionales de los medios. Esta tendencia amplía la vía de entrada a otras organizaciones que, aunque no dispongan de recursos para el marketing, sí son capaces de orientar su trabajo con medios a la incidencia y la difusión de conocimiento" (Gómez-Pérez et al., 2019, p. 47).

Este planteamiento, que probablemente beneficie a aquellas entidades con discursos poco hegemónicos, está relacionado con las redes sociales y su cada vez mayor relevancia a la hora de informarse.

La web 2.0 trajo consigo una gran oportunidad para las ONGs -sobre todo para aquellas de menor tamaño- puesto que permite

"alcanzar, sin necesidad de intermediación, sectores de población más alejados de su actividad" (Albaigès, 2012). Inicialmente la revolución digital parecía que se iba incluso a poder prescindir de los medios de comunicación conven- 
cionales y así apostar por un tipo diferente de comunicación directa entre ONGs y públicos. Sin embargo, por una parte, tal y como Gómez-Pérez et al. (2019) reconocen, el potencial de las redes sociales no se está aprovechando para generar y difundir discurso, sino que se usa para transmitir la actividad de la asociación en una sola dirección sin que haya conversación. Por otra parte, el peso de los medios de comunicación sigue siendo fundamental a la hora de conformar la agenda informativa y dotar de notoriedad y alcance a las ONGs y los movimientos sociales.

Ahora bien, es cierto que desde que las nuevas tecnologías permiten que los protagonistas hablen por sí mismos sin necesidad de intermediarios, la representación ha dejado de tener sentido. Jiménez-Gómez y Olcina-Alvarado (2017) opinan que, dado que a la hora de construir una noticia el periodista suele usar como fuentes de información a la persona afectada y al experto en el tema, las ONGs muchas veces no entran en ninguno de estos dos parámetros. Por ello, los testimonios se han convertido en un punto esencial para reestructurar la forma de actuar de la comunicación de las ONGs. En este sentido, Albaigès (2012) aboga por que las ONGs actúen como entidades facilitadoras de contacto directo entre ciudadanos y colectivos sociales, para que estos puedan transmitir sus historias. Cree que esta función puede ser un buen punto de partida para la innovación en el sector. Además de su credibilidad, no hay que olvidar que, en un contexto de crisis, las acciones de Relaciones Públicas permiten trabajar con un presupuesto limitado (Herrmann, 2004).

El contexto de crisis global vigente desde 2008 podría haber supuesto

"una oportunidad única para colocar nuevos mensajes en una sociedad potencialmente más receptiva a escuchar propuestas de cambio" (Santolino-Prieto, 2010, p. 233),

pero las ONGs no aprovecharon la situación y fueron los movimientos sociales quienes lo hicieron en su lugar. Como exponen Baamonde-Silva, Martínez-Rolán y Mínguez-González, las ONGs estuvieron ajenas a las experiencias de participación ciudadana que se dieron durante estos años y por ello el sector no fue

"un canal atractivo de movilización para los grupos sociales que tomaron la calle desde 2011" (Baamonde-Silva; Martínez-Rolán; Mínguez-González, 2016, p. 78).

El movimiento del $15 \mathrm{M}$ puso de manifiesto que la supuesta despolitización de la sociedad

"era tan sólo aparente y que el potencial de movilización social no estaba agotado" (Plataforma 2015 y más, 2011, p. 85).

Esto es algo que ya previeron Jerez-Novara, Sampedro y López-Rey, (2008): tras hacer un análisis histórico de las campañas de incidencia de las ONGs españolas, concluyendo que serían los movimientos sociales los que provocarían un replanteamiento del papel que juega la comunicación en el tercer sector. Por ello, muchas ONGs que hasta ese momento no se consideraban activistas, comenzaron a ofrecer a sus públicos un espacio en el que los mismos se convirtieran en protagonistas y parte activa de las causas por las que trabaja la organización (Soria-lbáñez, 2010).

Bacallao-Pino (2014) plantea que la comunicación tiene en los movimientos sociales un rol fundamental, ya que es el elemento que media en la dualidad propia de estos movimientos, caracterizada por enfrentar a la protesta (expresar el desacuerdo y cuestionar el orden social) con la propuesta (construir un modelo más justo a través de interrelaciones sociales).

No obstante, lo que según Nos-Aldás, Iranzo y Farné (2012) realmente define a los movimientos sociales actuales es su sinceridad comunicativa, siendo innovador el hecho de que pasaran del mensaje de intermediación propio de las ONGs ("nosotras lo hacemos por ti") a un discurso ligado al empoderamiento y a la responsabilidad individual, que invitaba a la acción colectiva ("actúa, a ti también te afecta").

Todos los autores comparten que la aparición y popularización de las redes sociales marcó un antes y un después en la comunicación de los movimientos sociales. Tanto Alonso-Muñoz (2016) como Haro-Barba y Sampedro-Blanco (2011) argumentan que éstos llegaron a unos niveles de fuerza y movilización desconocidos previamente en España gracias al surgimiento de la web 2.0. En este sentido, Castells (2009) vincula la democratización de las redes sociales y de los teléfonos móviles con el concepto de "autocomunicación de masas", el cual define como la comunicación de contenido autogenerado, emisión autodirigida y recepción autoseleccionada por las personas que comunican. Esta autocomunicación constituye, según él, una fuente de empoderamiento para la ciudadanía.

No obstante, como bien recuerda Fuchs (2014, en Alonso-Muñoz y Casero-Ripollés, 2016), el potencial de las redes sociales para introducir una demanda en la agenda ciudadana no debe sobredimensionarse, ya que éstas siguen siendo incapaces de llegar a la misma cantidad de público que los medios de comunicación convencionales.

Es el caso de Las Kellys, las cuales, aunque habían constituido grupos en Facebook en 2014, no empezaron a conocerse masivamente hasta su presentación pública en 2016, cuando poco a poco, gracias a que los medios de comunicación les empezaron a dar mayor cobertura, su caso entró en la agenda mediática y social.

\section{Objetivos y metodología}

Esta investigación plantea como objetivo general analizar la comunicación llevada a cabo por la organización Las Kellys como caso de estudio de un nuevo movimiento social cuyas prácticas de Relaciones Públicas con la prensa y en redes sociales pueden servir de referencia para la comunicación de otras organizaciones, especialmente ONGs. 
Como objetivos específicos, se plantea

- cuantificar la presencia de la temática de las condiciones laborales de las camareras de piso en los medios de comunicación digitales españoles;

- analizar el tratamiento de los medios de comunicación digitales españoles sobre la información acerca de las condiciones laborales de las camareras de piso y de las acciones realizadas por la asociación Las Kellys;

- determinar cuál ha sido el agente social que en mayor medida ha introducido el tema de las condiciones laborales de las camareras de piso en la agenda mediática;

- evaluar la estrategia de comunicación seguida por la asociación Las Kellys para introducir en la agenda mediática sus demandas laborales.

Las hipótesis de partida fueron que:

- la asociación Las Kellys ha introducido con mayor eficacia la problemática laboral de las camareras de piso en la agenda mediática española que otros agentes sociales;

- la estrategia comunicativa seguida por la asociación se ha basado en el empleo mayoritario de técnicas de storytelling y de la primera persona.

En cuanto a las técnicas de investigación empleadas, por un lado, se realizó un grupo de discusión el 28 de noviembre de 2018 en el que participaron tres representantes de Las Kellys Lanzarote, una representante de Las Kellys Madrid y un representante de Sindicalistas de Canarias, sindicato minoritario que presta ayuda al movimiento.

Por otro lado, se realizaron los tres tipos de entrevistas cualitativas descritos por Patton (2002):

En primer lugar, buscando un primer acercamiento al funcionamiento de la asociación Las Kellys, se hizo una entrevista conversacional informal a María del Mar Jiménez, una de las principales representantes y portavoces de Las Kellys Madrid.

Posteriormente, en enero de 2019, se realizó una extensa entrevista en profundidad basada en un guion a la presidenta de la asociación Las Kellys a nivel estatal Myriam Barros.

Por último, se realizaron entrevistas estandarizadas abiertas a cuatro periodistas de periódicos digitales españoles que habían redactado artículos sobre la asociación Las Kellys. Se buscó que la muestra fuese lo suficientemente representativa seleccionando a profesionales de medios de tendencias ideológicas diversas. Los periodistas entrevistados fueron

- Adrián Argudo Sánchez, redactor en El economista;

- Emilio J. Martínez Salazar, periodista en Eldiario.es Alicante;

- Pablo Rodero Sardinero, redactor de 20 minutos;

- Elena Berberana, periodista de Libertad digital (su caso es interesante ya que es la autora del único artículo identificado que critica abiertamente el trabajo de reivindicación de derechos laborales de la asociación Las Kellys).

El objetivo en este caso fue investigar la percepción que ellos tenían sobre la comunicación de Las Kellys y saber cómo fue su relación profesional con ellas.

Paralelamente, se llevaron a cabo tres análisis de contenido. En el primero de ellos se analizó el 100\% de los artículos redactados en castellano que incluyesen en su texto las palabras "camareras de piso" y/o "kellys" y que hubiesen sido publicados en medios de comunicación digitales españoles entre el 1 de septiembre de 2018 y el 28 de febrero de 2019. Se seleccionan estas fechas por ser un período de relativa calma mediática, posterior a la denominada "primera gran victoria de Las Kellys" (el reconocimiento de algunas de sus enfermedades profesionales acontecido el 31 de agosto de 2018) y previo al comienzo de la campaña electoral para las elecciones nacionales españolas ${ }^{1}$. Siguiendo los criterios de la muestra, se detectó y analizó un total de 553 publicaciones en 156 medios de comunicación digitales.

A partir de ese primer análisis de contenido se identificaron las tres delegaciones de Kellys con mayor presencia mediática (las de Lanzarote, Madrid y Barcelona, por este orden) y se hizo un segundo análisis de contenido cuya muestra fue el $100 \%$ de los posts publicados entre el 1 de enero y el 28 de febrero de 2019 en las páginas de Facebook de estas tres delegaciones de la asociación. Se seleccionaron dichas fechas por ser los dos meses más recientes que coincidiesen con las fechas de la muestra del análisis de contenido de las noticias en medios digitales. Al ser los más recientes, se facilitó el acceso a las publicaciones. Asimismo, se seleccionó la red social Facebook por ser ésta, según las entrevistas realizadas, la que la asociación Las Kellys utiliza más para comunicarse.

Por último, se realizó un tercer análisis de contenido cuya muestra fueron los quince vídeos con mayor número de visualizaciones a fecha del 11 de junio de 2019 del canal de YouTube de la asociación Las Kellys.

http://bit.ly/2IrINvE

\section{Resultados cualitativos: "Yo me limito a contar mi historia"}

Una vez realizadas las entrevistas y el focus group procedimos a plantear los principales resultados obtenidos centrándonos, en primer lugar, en el análisis global de las opiniones de las representantes del movimiento Las Kellys, quienes declararon que tanto expresarse en primera persona como relatar sus historias personales son aspectos que ellas consideran clave en el éxito de su comunicación. 
En cuanto a lo primero, la propia presidenta de la asociación señaló que aprueban que otras personas les ayuden y les den herramientas de comunicación, pero que no quiere que hablen por ellas ni que las representen:

"Yo acepto que el abogado me acompañe a los actos, pero quien habla soy yo".

En la misma línea, una representante de Las Kellys Lanzarote indicó en el focusgroup:

"Hablamos en primera persona, de lo que nos afecta [...]. Hablamos tal y como somos, sin tecnicismos".

Tanto ella como varias de sus compañeras consideran que este tipo de lenguaje les ayuda a captar la atención y generar simpatía:

"Lo que pasa es que la gente valora que usemos una comunicación que se entienda. Porque hay mucha gente que va a una charla y te suelta un rollo institucional. Nosotras en cambio usamos el lenguaje de la calle, el cercano, que involucra y que implica".

Precisamente los periodistas en las entrevistas valoran positivamente el lenguaje informal de Las Kellys:

"Su forma de comunicación no es profesional. Usan una comunicación completamente natural. Cuando se profesionaliza la comunicación, puede tener un impacto más automático a través de notas de prensa o publirreportajes como lo hacen las ONGs, pero lograr un apoyo solidario como el que han conseguido Las Kellys es complicado si no se hace una comunicación digamos humana, sin esa barrera de prototipos de mensaje ya estandarizado que normalmente recibes de gabinetes de comunicación. Esta gente es no profesional y sus mensajes son más naturales. Creo que esa es un poco la diferencia".

Sobre el uso de la narrativa, varias kellys afirman que contar sus historias de explotación laboral es algo que les parece muy importante, ya que es una forma de evitar que se les note que la mayoría de las camareras de piso son personas sin estudios y sin facilidad de oratoria. Por eso, han determinado la siguiente estructura de portavocía: quienes quieren hablar en nombre del colectivo han de formarse (con ayuda de pequeños sindicatos que las asesoran y de documentos que se comparten entre ellas en los grupos de WhatsApp y Telegram que tienen para coordinarse), y quienes no están formadas hablan únicamente a nivel individual sobre sus historias personales. Por ejemplo, una representante de la delegación madrileña de Las Kellys afirmó:

"Yo me limito a contar mi historia. No cuento nada más porque yo no entiendo y no quiero por error poner en mal lugar a las compañeras o hablar de algo que no sé".

Los periodistas entrevistados aprecian la posibilidad de transmitir relatos individuales:

"Todas te cuentan su historia personal y eso es algo que para los medios de comunicación es muy importante, porque aquí hay una disputa entre los periodistas de buscar historias personales y ellas representan cada una una historia de sufrimiento personal diferente. A nivel mediático eso funciona. Vender historias personales - no grupales-y poner cara al problema es la clave".

Además de eso, la periodista Elena Berberana indica que a Las Kellys también le favorece su naming:

"Si tú consigues una etiqueta que funcione en los medios de comunicación, vas a triunfar a nivel de impacto mediático".

Por otro lado, en relación con las estrategias de Relaciones Públicas que el movimiento de camareras de piso ha empleado para salir en los medios, la presidenta de Las Kellys explicó que al principio se esforzaban para captar la atención de los periodistas subiendo a sus redes sociales imágenes y vídeos caseros que sabían que iban a ser llamativos para que se compartiesen. Una vez alcanzado el interés mediático, en la actualidad no lanzan apenas notas de prensa, sino que son los periodistas quienes las llaman:

"Ahora, cada vez que sale una noticia de algún asunto laboral en el sector del turismo se llama a los sindicatos, pero también se nos llama a nosotras para ver qué tenemos que decir".

Todos los periodistas consultados dan fe de que fueron ellos quienes dieron el primer paso y se acercaron al movimiento, atraídos por su estilo innovador y autoorganizado de reivindicación sindical. Asimismo, todos valoran positivamente la facilidad de contacto, a través de la cual consiguieron de manera rápida y eficaz las declaraciones de alguna de las representantes de la organización.

\section{Análisis de contenido: personalización y primera persona}

Como se ha mencionado previamente, en los seis meses del análisis de contenido se detectaron y analizaron 553 publicaciones que cumpliesen los criterios de la muestra. Estos artículos estuvieron presentes en 156 medios de comunicación digitales. Esta cobertura fue notablemente amplia.

De los medios que compusieron la muestra, el que más artículos publicó fue Eldiario.es, con cuarenta noticias: un 7\% del total de 553 publicaciones. No obstante, como se observa en la figura 1, la categoría que predomina es, con diferencia, la de "medios autonómicos" (237 publicaciones, el 43\% de la muestra). En segundo lugar, queda la categoría de "medios de tendencia política de izquierdas", con un $15 \%$. 
El agente social que protagoniza el $32 \%$ de las publicaciones de la muestra es la asociación Las Kellys o representantes de ésta, quedando en primera posición frente a otros agentes. Como se observa en la figura 2, los partidos u organismos políticos son los protagonistas del $16 \%$ de la muestra y las camareras de piso (sin vinculación explícita a Las Kellys dentro del texto del artículo), del 15\%. 'Sector turístico' y 'Otros colectivos en lucha' empatan a un $8 \%$ y los sindicatos quedan en última posición con un 3\% del total.

La fuente principal de información en el $24 \%$ de las publicaciones fueron Las Kellys, por delante de los sindicatos $(21 \%)$ y de los partidos $u$ organismos políticos (19\%). Sin embargo, tal y como se observa en la figura 3, la categoría que queda en primera posición es 'Otros'. Es de señalar que dentro de esta categoría se incluyen artículos con gran variedad de fuentes, entre ellos aquellos cuya fuente principal ha sido el o la periodista del medio haciendo investigación propia. Estos datos corroboran la hipótesis de que la asociación Las Kellys ha introducido con mayor eficacia la problemática laboral de las camareras de piso en la agenda mediática española que otros agentes sociales.

En cuanto a la presencia en el artículo de la palabra "kellys" o "camareras de piso" (ya sea en singular o en plural), el $51,18 \%$ de las publicaciones contiene ambos términos; el $33,27 \%$, únicamente "camareras de piso"; y el $15,55 \%$ restante, únicamente "kellys". El hecho de que el $66,73 \%$ de la muestra use la palabra "kellys" valida la idea de la periodista Elena Berberana de que el naming del movimiento funciona mediáticamente.

En el 20\% de los artículos de la muestra alguien utiliza la primera persona del singular o del plural para expresarse. Esta cifra sube al 59\% cuando la fuente del artículo son kellys. Se reduce a $12 \%$ cuando dicha fuente son los sindicatos y al $5 \%$ cuando se trata de partidos u organismos políticos.

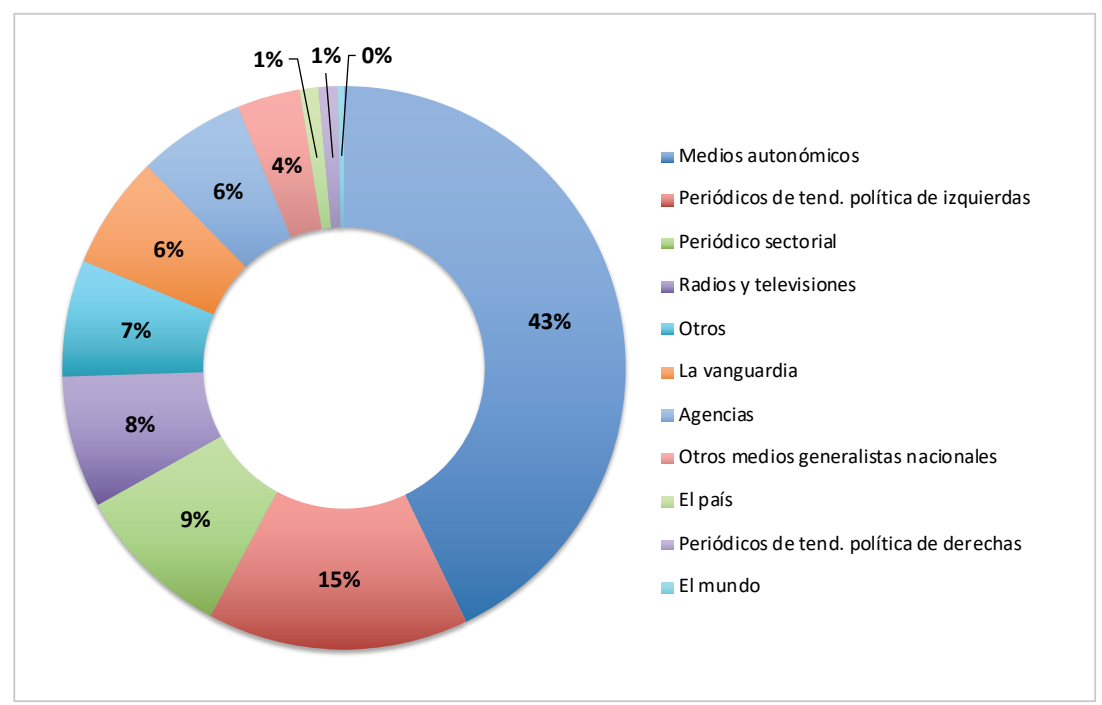

Figura 1. Porcentaje de publicaciones por medio de comunicación

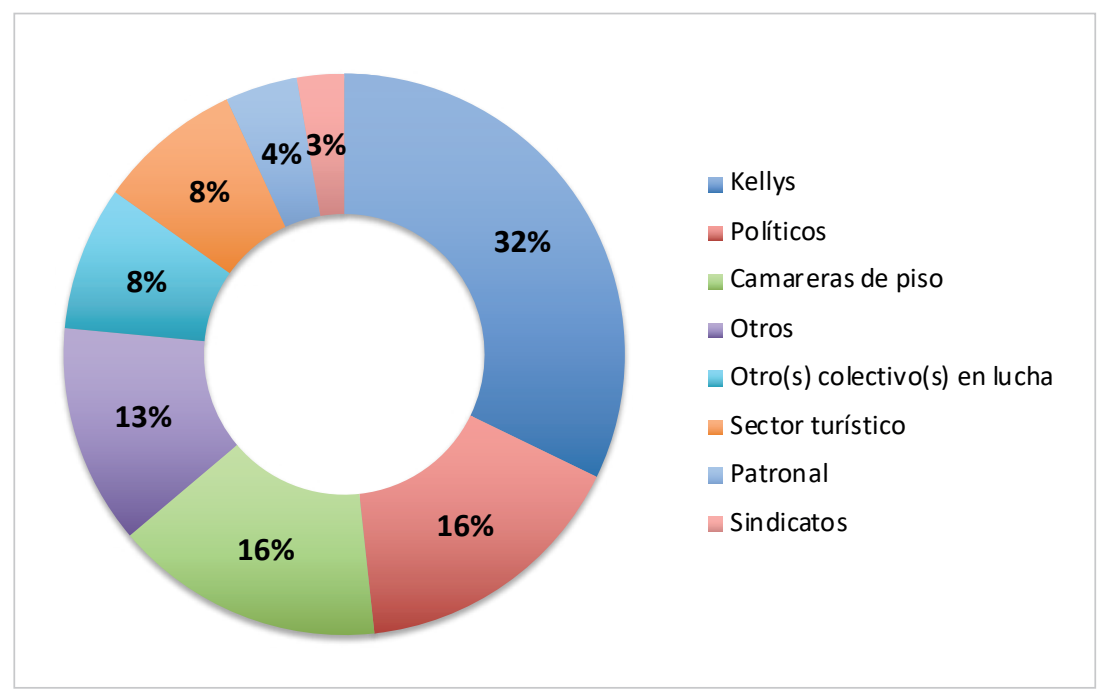

Figura 2. Porcentaje de publicaciones por protagonista del artículo

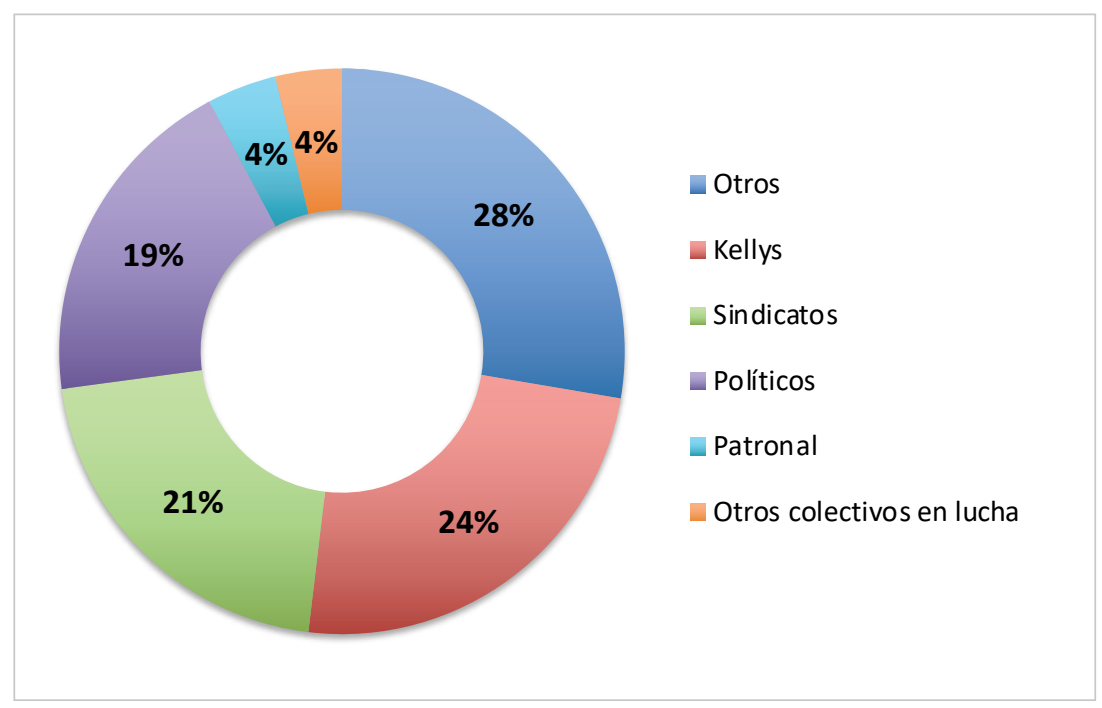

Figura 3. Porcentaje de publicaciones por fuente de información

Algo similar ocurre al tener en cuenta el uso del storytelling dentro de las publicaciones. En el 15\% de la muestra se emplean técnicas de narración. El porcentaje asciende a 34\% cuando la fuente principal del artículo son kellys. Baja al 14\% cuando la fuente son los sindicatos y al $2 \%$ cuando son partidos u organismos políticos, tal y como se indica en la figura 4. 
Por lo que respecta al análisis de contenido de los 246 posts publicados entre enero y febrero de 2019 en las páginas de Facebook de Las Kellys Madrid, Las Kellys Barcelona y Las Kellys Lanzarote, en el $51 \%$ de las publicaciones se emplea la primera persona del singular o del plural para expresarse. En cambio, las técnicas de storytelling sólo se utilizan en el $10 \%$ de la muestra.

Por último, según el análisis de contenido de las piezas audiovisuales, Las Kellys utilizan la primera persona del singular o del plural para expresarse en el $73,3 \%$ de los quince vídeos analizados. En cambio, sólo en el $46,7 \%$ de la muestra se utilizan técnicas de storytelling.

Según los datos expuestos, resumidos en la figura 5 , únicamente podemos corroborar una parte de la segunda hipótesis inicial, en el sentido de que la estrategia comunicativa seguida por la asociación Las Kellys sí que se ha basado en el empleo mayoritario de la primera persona, pero no tanto de las técnicas de storytelling.

\section{Aportaciones para la comunicación de las ONGs}

Los resultados obtenidos pueden servir como referencia para la práctica profesional de Relaciones Públicas de los departamentos de comunicación de las ONGs. Varios autores han identificado diferentes necesidades comunicativas en las ONGs que la estrategia de comunicación de los movimientos sociales en general y, nos permitimos añadir que de Las Kellys en particular, quizá puede ayudar a satisfacer.

En primer lugar, como ya se ha señalado, autores como Santolino-Prieto (2010) o la Plataforma 2015 y más (2011) indican que muchas ONGs no encuentran el tono adecuado para hacer llegar de forma sencilla la complejidad de sus programas y de las problemáticas que abordan, lo cual tiene como consecuencia que se crea un gap entre lo que estas entidades hacen y lo que comunican. En cambio, Las Kellys confían en la capacidad de comprensión del público y, para hacer llegar su mensaje, usan un lenguaje cercano y coloquial, evitando los tecnicismos.

Sampedro, Jerez-Novara y López-Rey (2002), Pagola-Carte (2009) y Chaves-Gil (2012) consideran que la forma de actuar de los medios de comunicación obliga a las ONGs a seguir un estilo comunicativo marcado por mensajes llamativos y sencillos. Las Kellys también utilizan dicho estilo, pero lo complementan con la transmisión de información a través de historias personales. Como se ha demostrado, esto es algo que agentes sociales como partidos políticos o sindicatos hacen en mucha menor medida cuando hablan del mismo tema. Además de eso, Las Kellys también buscan ser lo suficientemente diferentes e innovadoras en su forma de actuar, con el objetivo de que sus acciones den que hablar y sean los periodistas quienes acudan a ellas para obtener información.

Por último, Albaigès (2012) detecta que el auge de las redes sociales ha ocasionado que la costumbre de la comunicación de las ONGs de representar la voz de personas desfavorecidas haya dejado de tener sentido. Las Kellys se adaptan al nuevo paradigma hablando en primera persona y promoviendo que cada camarera de piso se atreva a narrar sus experiencias. Del mismo modo, las ONGs podrían establecer vías formativas para que los beneficiarios de sus programas de ayuda quieran contar sus historias, de tal manera que la entidad se convirtiese en un canal para la transmisión de testimonios de personas en situación de vulnerabilidad. 


\section{Conclusiones}

A lo largo de los últimos años, el colectivo de Las Kellys ha logrado visibilizar la explotación laboral que afecta a las camareras de piso e introducir en el debate político y social español la cuestión de la calidad del trabajo en el sector turístico, utilizando eficazmente las relaciones con los medios de comunicación y su participación en redes sociales. De esta forma, tal y como se ha comprobado, Las Kellys han sido el agente social que en mayor medida ha introducido la problemática de las camareras de piso en la agenda mediática digital española, por encima de los sindicatos u otros agentes sociales.

Para conseguir esto, la estrategia de comunicación empleada por la organización Las Kellys se ha basado en el empleo mayoritario de la primera persona, aunque no tanto en el uso del storytelling. El relativo uso a nivel cuantitativo de las estrategias narrativas choca con las declaraciones de las representantes del movimiento en las entrevistas y focus group, donde afirmaron que relatar sus historias personales es un aspecto que ellas consideran fundamental en su comunicación. Dado que estas narrativas son bien valoradas por los periodistas y tienen significativos resultados en redes sociales, es una herramienta comunicativa a la que las ONGs deberían prestar atención y seguramente potenciar.

Las Kellys usan un lenguaje cercano y coloquial, un estilo comunicativo sencillo y llamativo empleando historias personales llenas de verdad. El caso de Las Kellys demuestra la comprensión de que, en el escenario digital y mediático actual, es necesaria la adaptación al nuevo paradigma comunicativo a través de acciones de Relaciones Públicas que impliquen un diálogo sincero con los medios de comunicación y en las redes sociales que apueste por la autenticidad de las narrativas en primera persona, lo que sin duda puede ser una lección de interés para dotar de mayor credibilidad, cercanía y autenticidad determinadas acciones de comunicación de las ONGs, que tienen entre sus socios y los beneficiarios de sus programas de ayuda un activo de comunicación potencial a través de la narración auténtica de sus historias en primera persona.

\section{Notas}

1. El 5 de marzo de 2019 se publicó en el Boletín oficial del Estado el real decreto de disolución de las Cortes y de convocatoria de elecciones.

\section{Bibliografía}

Albaigès, Jaume (2012). “¿Cómo generar conversaciones y promover la participación desde las ONGD?”. En: Ramil-Ferreiro, Xosé (coord.). ParadigmáTIC@s. Comunicación y cultura digital en las ONG de desarrollo. Madrid: Coordinadora de ONG para el Desarrollo-España, pp. 62-68.

https://desycom.files.wordpress.com/2012/02/culturadigital.pdf

Alonso-Muñoz, Laura (2016). “Estrategia comunicativa de la Plataforma de Afectados por la Hipoteca en las redes sociales”. Revista de la Asociación Española de Investigación de la Comunicación, v. 3, n. 5, pp. 42-53.

http://revistaeic.eu/index.php/raeic/article/view/119

Alonso-Muñoz, Laura; Casero-Ripollés, Andreu (2016). "La influencia del discurso sobre cambio social en la agenda de los medios. El caso de la Plataforma de Afectados por la Hipoteca”. Obets: Revista de ciencias sociales, v. 11, n. 1, pp. 25-51.

https://doi.org/10.14198/OBETS2016.11.1.02

Arroyo-Almaraz, Isidoro; Baladrón-Pazos, Antonio J.; Martín-Nieto, Rebeca (2013). “La comunicación en redes sociales: Percepciones y usos de las ONG españolas". Cuadernos.info, n. 32, pp. 77-88.

https://doi.org/10.7764/cdi.32.497

Arroyo-Almaraz, Isidoro; Calle-Mendoza, Samuel; Van-Wyk, Cliff (2018): "La eficacia en la comunicación de las ONGD. El uso de Facebook en campañas de emergencia”. Revista latina de comunicación social, n. 73, pp. 765-789.

http://www.revistalatinacs.org/073paper/1281/40es.html

Baamonde-Silva, Xosé; Martínez-Rolán, Xabier; Mínguez-González, María-Isabel (2016). "Las ONG como agentes de transformación social. Del asistencialismo a la movilización”. Obets: Revista de ciencias sociales, v. 11, n. 1, pp. 75-96. https://doi.org/10.14198/OBETS2016.11.1.04

Bacallao-Pino, Lázaro M. (2014). “Comunicación contrahegemónica, movimientos sociales y cambio social”. En: Gallardo-Vera, Luis (ed.). Nuevos movimientos sociales y comunicación corporativa: la revolución de la acción. La Plata: Ediciones de Periodismo y Comunicación, pp. 122-141. ISBN: 9789503411490

https://perio.unlp.edu.ar/iicom/sites/perio.unlp.edu.ar.iicom/files/nuevos_movimientos_sociales_y_comunicacion_ corporativa.pdf 
Balas-Lara, Monserrat (2008). “El reto de la comunicación en el tercer sector no lucrativo". Revista española del tercer sector, v. 8, pp. 17-38.

https://dialnet.unirioja.es/servlet/articulo?codigo=2649005

Balas-Lara, Monserrat (2012). La gestión de la comunicación en el tercer sector: Cómo mejorar la imagen de las ONG. Pozuelo de Alarcón: Esic. ISBN: 9788473568074

Barranquero-Carretero, Alejandro (2014). “Comunicación, cambio social y ONG en España. Pistas para profundizar en la cultura de la cooperación desde los nuevos movimientos comunicacionales. El caso del 15M". Commons: revista de comunicación y ciudadanía digital, v. 3, n. 1, pp. 6-28.

http://hdl.handle.net/10016/20699

Benet, Vicente J. (2003). “El espectáculo solidario: la publicidad en el tercer sector y su proyección cultural”. En: Benet, Vicente J.; Nos-Aldás, Eloías (eds.). La publicidad en el tercer sector. Tendencias y perspectivas de la comunicación solidaria. Capellades: Icaria Editorial, pp. 15-52. ISBN: 9788474266801

Cañada, Ernest (2014). "Las que limpian los hoteles". El país, 30 julio.

https://elpais.com/elpais/2014/07/30/alterconsumismo/1406706574_140670.html

Cañada, Ernest (2015). Las que limpian los hoteles. Historias ocultas de precariedad laboral. Icaria Editorial. ISBN: 978 8498886849

Castells, Manuel (2009). Comunicación y poder. Madrid: Alianza Editorial. ISBN: 9788420684994

Castillo-Esparcia, Antonio (2007). "Relaciones públicas en las organizaciones no gubernamentales". Sphera pública, v. 7, pp. 193-210.

https://dialnet.unirioja.es/servlet/articulo?codigo=2510531

Chaves-Gil, Iñaki (2012). “Comunicación y sociedad civil. El caso de las ONGD”. En: Chaves-Gil, Iñaki (coord.). Comunicación para el cambio social. Universidad, sociedad civil y medios. Madrid: Catarata, pp. 67-83. ISBN: 9788483197547

Díez-Rodríguez, Ángeles (2002). “Las ONG como campo de relaciones sociales”. En: Revilla-Blanco, Marisa (ed.). Las ONG y la política. Madrid: Istmo, pp. 120-171. ISBN: 8470904043

Erro-Sala, Javier (2003). Descubrir y construir procesos de comunicación social. Aportes para diseñar políticas, estrategias y estructuras de comunicación en las ONGD. Bilbao: Hegoa.

http://publicaciones.hegoa.ehu.es/uploads/pdfs/54/Descubrir_y_construir_procesos_de_comun_social.pdf?1488539187

Gálvez-Rodríguez, María-del-Mar; Caba-Pérez, María-del-Carmen; López-Godoy, Manuel (2009). “La transparencia on-line de las ONG españolas". Revista española del tercer sector, n. 13, pp. 63-88.

https://dialnet.unirioja.es/servlet/articulo?codigo $=3102628$

Gómez-Gil, Carlos (2005). Las ONG en España: de la apariencia a la realidad. Madrid: La Catarata. ISBN: 8483192217

Gómez-Pérez, José-Manuel; Jiménez-Rivero, Matilde; Rosado-Morón, Itziar; Sánchez-Jacob, Eduardo (2019). Nadie dijo que fuera fácil. Un relato colectivo, optimista y cuestionador sobre cómo las ONGD implicamos a la ciudadanía. Madrid: Ongawa. ISBN: 9788461742004

https://ongawa.org/nadie-dijo-facil-resumen

González Luis, Hildegart (2006). Estrategias de comunicación en las ONG de desarrollo: departamentos, funciones e impacto en los medios. Madrid: Cideal. ISBN: 8487082319

Haro-Barba, Carmen; Sampedro-Blanco, Víctor (2011). “Activismo político en Red: del Movimiento por la Vivienda Digna al 15M". Teknokultura, v. 8, n. 2, pp. 157-175.

https://revistas.ucm.es/index.php/TEKN/article/view/48025

Herrmann, Frank (2004). Relaciones públicas para ONG. Konrad Adenauer-Stiftung.

Jarque-Balaguer, Marta (2018). La explotación laboral en el sector hostelería y turismo. Trabajo fin de grado inédito. Universitat Jaume I.

http://repositori.uji.es/xmlui/handle/10234/177306

Jerez-Novara, Ariel; Sampedro, Víctor; López-Rey, José (2008). Del 0,7\% a la desobediencia civil. Política e información del movimiento y las ONG de desarrollo (1994-2000). Madrid: Centro de Investigaciones Sociológicas. ISBN: 97884 74764550

Jiménez-Fernández, María-Isabel; Cabanillas, Juan-Luis; Quintana-Betancor, Alejandro; Martín-de-la-Coba Inmaculada (2010). "Lesiones osteomusculares en camareras de piso del sector hostelería canario". Revista digital de prevención, n. 2, pp. 1-20.

http://rabida.uhu.es/dspace/handle/10272/5471 
Jiménez-Gómez, Isidro; Olcina-Alvarado, Mariola (2017). Manual de comunicación para la ciudadanía organizada. Madrid: Libros en acción. ISBN: 9788494615177 http://manualdecomunicacion.org/descargalibro

Mantovano, Patricia (2015). Dolor crónico por trastornos musculo-esqueléticos (TME), síntomas de ansiedad y depresión. Estudio descriptivo de la situación de las camareras de pisos en España. España: UITA-Uthgra. https://www.ccoo-servicios.es/archivos/hosteleria/Estudio-Camareras-Espana-AUITA-CCOO.pdf

Marí-Sáez, Víctor-Manuel (2017). “Historia de la comunicación para el desarrollo y el cambio social en España”. Telos, n. 106, pp. 27-38.

https://telos.fundaciontelefonica.com/archivo/numero106/marginalidad-institucionalizacion-e-implosion-de-uncampo-en-crecimiento

Marín-Dueña, Pedro-Pablo; Gallardo-Vera, Luis (2014). "El marketing y la comunicación corporativa en el tercer sector". En: Gallardo-Vera, Luis (ed.). Nuevos movimientos sociales y comunicación corporativa: la revolución de la acción. La Plata: Ediciones de Periodismo y Comunicación, pp. 84-107.

https://libros.unlp.edu.ar/index.php/unlp/catalog/book/186

Martínez-Osés, Pablo J. (2011). “Redefinición del papel de las ONG: hacia una mirada más política”. En: Jornadas eficacia del desarrollo y ONGDs: renovando nuestro papel. Madrid: Plataforma 2015 y más.

https://bit.ly/2ES48gb

Nos-Aldás, Eloísa; Iranzo, Amador; Farné, Alessandra (2012). "La eficacia cultural de la comunicación de las ONGD: los discursos de los movimientos sociales actuales como revisión”. CIC. Cuadernos de información y comunicación, v. 17, pp. 209-232.

https://doi.org/10.5209/rev_CIYC.2012.v17.39265

Nos-Aldás, Eloísa; Santolino-Prieto, Monserrat (2015). “La investigación en comunicación y cooperación en los nuevos escenarios de movilización social: ONGD, objetivos de justicia social y eficacia cultural". Revista de la Asociación Española de Investigación de la Comunicación, v. 2, n. 4, pp. 1-7.

http://www.revistaeic.eu/index.php/raeic/article/view/50/47

Pagola-Carte, Juan (2009). Comunicación para el desarrollo: la responsabilidad en la publicidad de las ONGD. San Sebastián: GizaEskubideak. ISBN: 9788461328673

Patton, Michael Q. (2002). Qualitative evaluation and research methods. Thousand Oaks, California: Sage. ISBN: 9780 803937796

Plataforma 2015 y más (2011). “Renovando nuestro papel hacia la transformación social”. En: Martínez, Ignacio (coord.). Renovando el papel de las ONGD: hacia la transformación social. Madrid: Editorial 2015 y más, pp. 69-90. http://www.2015ymas.org/IMG/pdf/Renovando_el_papel.pdf

Sampedro, Víctor; Jerez-Novara, Ariel; López-Rey, José (2002). “ONG, medios de comunicación y visibilidad pública. La ciudadanía ante la mediatización de los mensajes sociales”. En: Revilla-Blanco, Marisa (ed.). Las ONG y la política. Madrid: Istmo, pp. 251-285. ISBN: 8470904043

Santolino-Prieto, Montse (2010). "Recuperando la esencia: las ONGD como agentes de comunicación para el cambio social”. En: Burgui, Teresa; Erro, Javier (coords.). Comunicando para la solidaridad y la cooperación. Cómo salir de la encrucijada. Pamplona: Foro Comunicación, Educación y Ciudadanía, pp. 221-256.

https://plataformavoluntariado.org/wp-content/uploads/2018/10/recuperando-la-esencia-las-ongd-como-agentes-decomunicacion-para-el-cambio-social.pdf

Soria-Ibáñez, María-del-Mar (2010). "El ciberactivismo, nuevo modelo de Relaciones Públicas en las ONGs". Icono 14, v. 8, n. 3, pp. 288-302.

https://doi.org/10.7195/ri14.v8i3.242

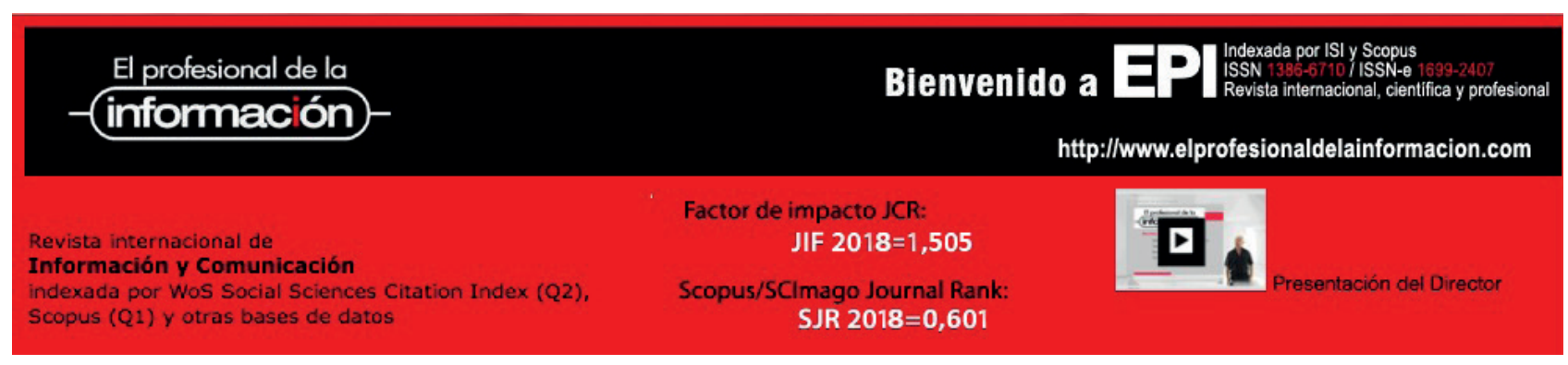

